\title{
Development and Testing of a Methane/Oxygen Catalytic Microtube Ignition System for Rocket Propulsion
}

This study sought to develop a catalytic ignition advanced torch system with a unique catalyst microtube design that could serve as a low energy alternative or redundant system for the ignition of methane and oxygen rockets. Development and testing of iterations of hardware was carried out to create a system that could operate at altitude and produce a torch. A unique design was created that initiated ignition via the catalyst and then propagated into external staged ignition. This system was able to meet the goals of operating across a range of atmospheric and altitude conditions with power inputs on the order of 20 to 30 watts with chamber pressures and mass flow rates typical of comparable ignition systems for a $100 \mathrm{lbf}$ engine. 
Development and Testing of a Methane/Oxygen Catalytic Microtube Ignition System for Rocket Propulsion

\section{$48^{\text {th }}$ AIAA/ASME/SAE/ASEE Joint Propulsion \\ Conference and Exhibit July 31, 2012}

Matthew Deans, Steven Schneider

(Presented by: William Marshall)

NASA Glenn Research Center

Cleveland, $\mathrm{OH}$ 


\section{Overview}

- This study developed a catalytic ignition torch system using catalyst microtubes

- The goal was to develop a torch igniter similar to traditional spark systems for a $100 \mathrm{lbf}$ engine with reduced power requirement compared to the current state of the art of $70 \mathrm{~W}$ needed applied to the exciter of comparable spark hardware

- A system was developed using up to $30 \mathrm{~W}$ that was able to ignite and create a torch

- Breakdown

- Background/Previous Work

- Facility

- Catalyst

- Catalyst at Atmospheric Pressure Conditions

- Catalyst at Altitude Pressure Conditions

- Staging

- Primary Augmenter at Altitude Pressure Conditions

- Secondary Augmenter at Altitude Pressure Conditions

- Main Torch at Atmospheric Pressure Conditions

- Conclusions

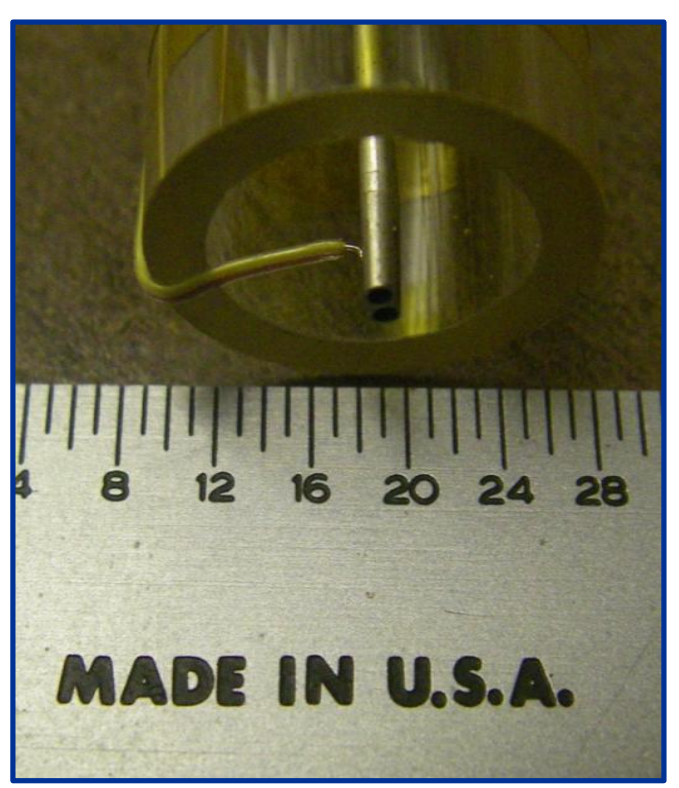




\section{Background/Previous Work}

- The Propulsion and Cryogenics Advanced Development (PCAD) project sought to develop enabling technologies for high performing non-toxic propulsion systems

- The project sought to reduce risk for Methane/Oxygen propulsion systems, develop alternative and redundant technologies

- As compared to Hydrogen/Oxygen and hypergols in use, Methane/Oxygen has greater ignition challenges; higher initial energy requirements, longer ignition delay

- Catalysts have the natural ability to lower ignition energy requirements

- Current catalysts used are typically packed beds or monoliths

- Large mass causing poor thermal response

- Injection challenges

- Non-uniformities in the bed

- Multiple flow paths

- High pressure drops

- Restricted by the limiting temperature of the substrate

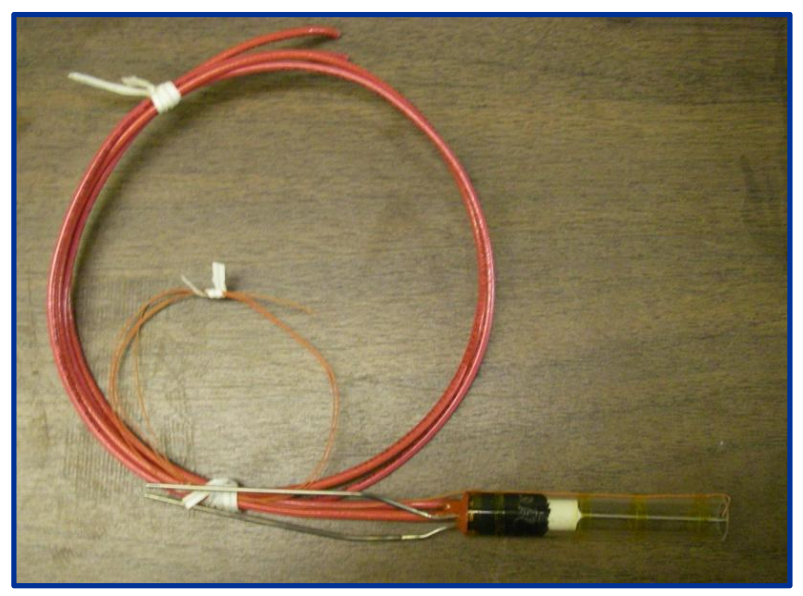

- Using pure metal catalyst microtubes would potentially allow for the negation of these drawbacks

- Catalytic microtubes have been previously studied for micropropulsion and microcombustor applications, not largely implemented or applied to ignition 


\section{Facility}

- Testing was carried out in the Research Combustion Laboratory (RCL) test cell 21 at NASA Glenn Research Center

- Capable of testing at both atmospheric and altitude conditions

- Controlled by an air driven ejector system with suction capacity of approximately $0.12 \mathrm{lbm} / \mathrm{s}$ dry air equivalent (DAE)

- Able to reduce the pressure within the test chamber to a steady value of approximately 5 torr $(0.1$ psia)

- Valves and data system are controlled via a programmable logic controller (PLC) and a graphical user interface

- Data is acquired at a variable rate, typically 10 to $50 \mathrm{~Hz}$ though the sampling rate can be raised higher

- Post processing of raw data is conducted on PCs by user written code

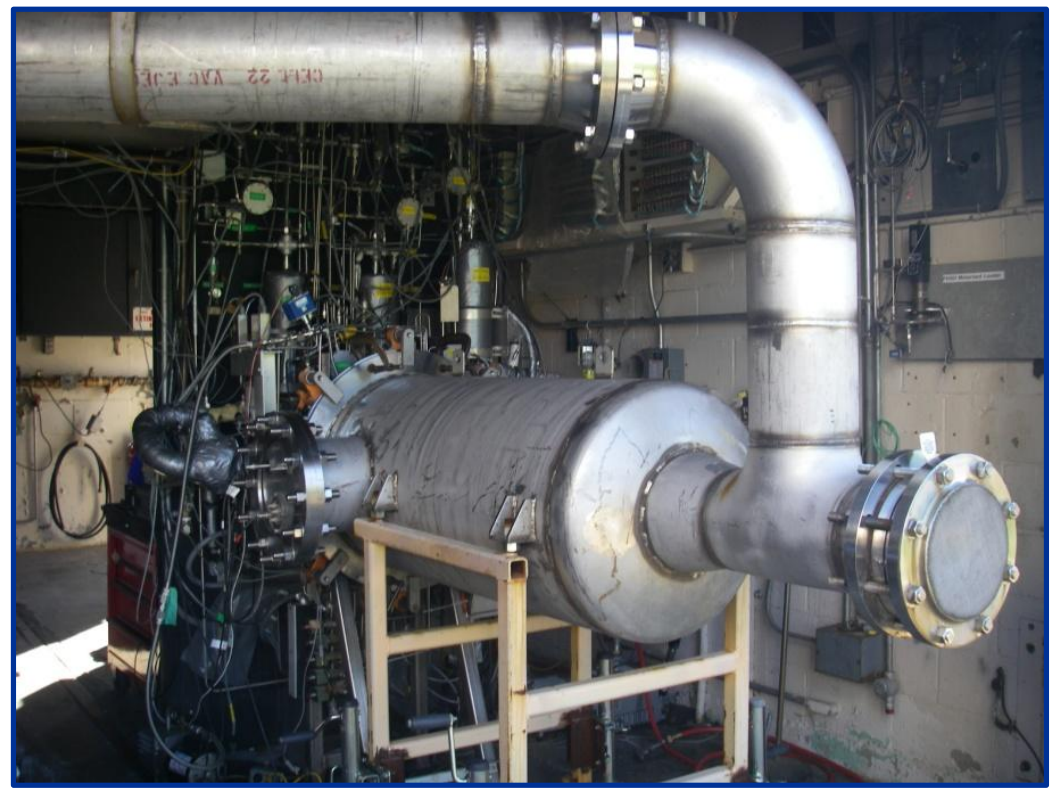




\section{Catalyst}

- Testing of other microtube configurations with preheated gas showed that heat losses from the tubes were a large challenge leading to a resistively heated element instead

- Prior testing with single tubes showed that the hottest point with direct electrical application occurred in the middle of the catalyst tube's electrical path; losses to electrical connections were large

- This unique configuration has two catalyst tubes welded together at the tips and electrically attached near the gas inlets so the hottest point at the middle of the electrical path is at the flow exit; 'wishbone' configuration

- Tubes are drawn platinum, $0.8 \mathrm{~mm} \mathrm{ID,} 1.0 \mathrm{~mm}$ OD

- $10 \mathrm{~cm}$ long, though, with the fittings, electrical attachments, and the weldment, the effective heated length of each tube is $\sim 8.0 \mathrm{~cm}$ long

- Shrouded in quartz tube to allow visibility and cantilevered from base to maintain thermal and electrical isolation

- Designed for flows on order of $0.01 \mathrm{~g} / \mathrm{s}$; previously modeled and shown to cause ignition with desired power input range, $\sim 1 \%$ of main igniter flows

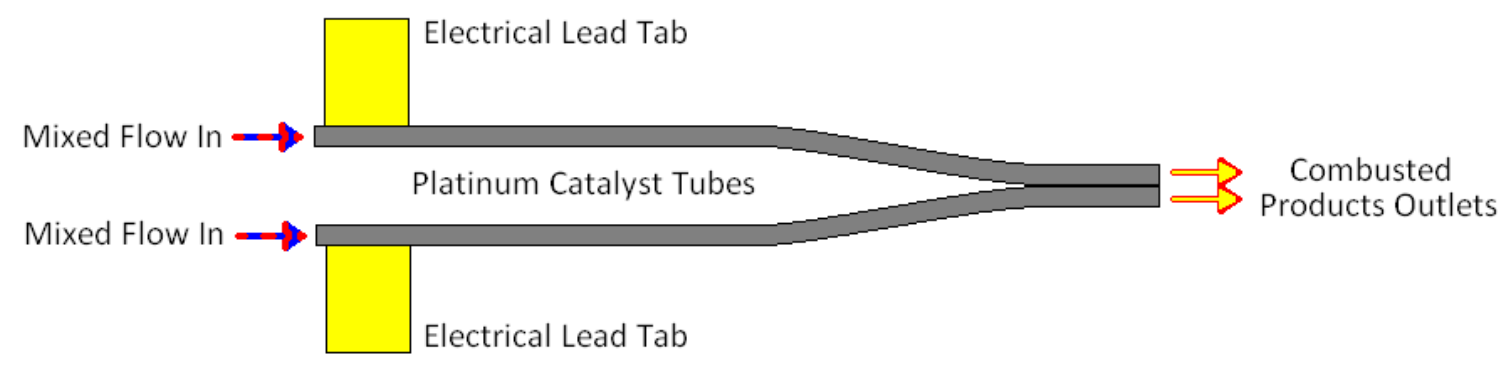




\section{Catalyst at Atmospheric Pressure Conditions}

- Ignition was demonstrated with pre-mixed gas through a range of mass flow rate and mixture ratio variation $(\mathrm{O} / \mathrm{F}=1.0 \& 1.66)$

- Catalyst was preheated for $30 \mathrm{sec}$, then flow initiated

- Applying more current to catalyst increases the preheat temperature

- Though there are different temperature trends shown for different mixture ratios, this is a result of different preheating scheme

- For the same mass flow rate and different mixture ratio there is a similar increase in temperature upon ignition

- For varying flow rate, tip temperature increases with flow rate; due to location of flame kernel
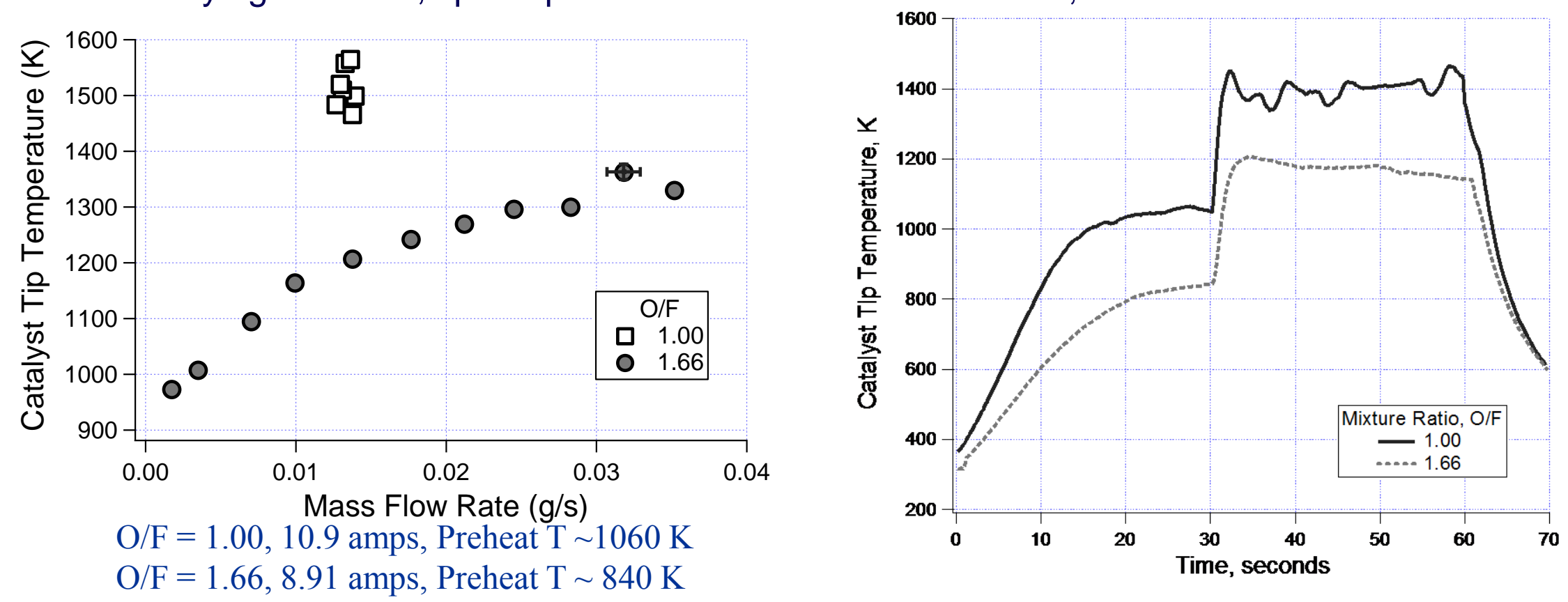


\section{Catalyst at Altitude Pressure Conditions}

- Tests were conducted over a range of flow rate and mixture ratios $(\mathrm{O} / \mathrm{F}=0.9,1.0 \& 1.66)$

- Post ignition temperature rise is a similar order of magnitude to atmospheric tests

- The lowest $\mathrm{O} / \mathrm{F}$ mixture ratios trended similarly, with the $\mathrm{O} / \mathrm{F}=1$ mixture being marginally hotter through the regime

- Though $\mathrm{O} / \mathrm{F}=1.66$ should have a hotter gas phase flame, the ignition kernel appeared earlier within the catalyst and required more flow to drive it away from the heat sink
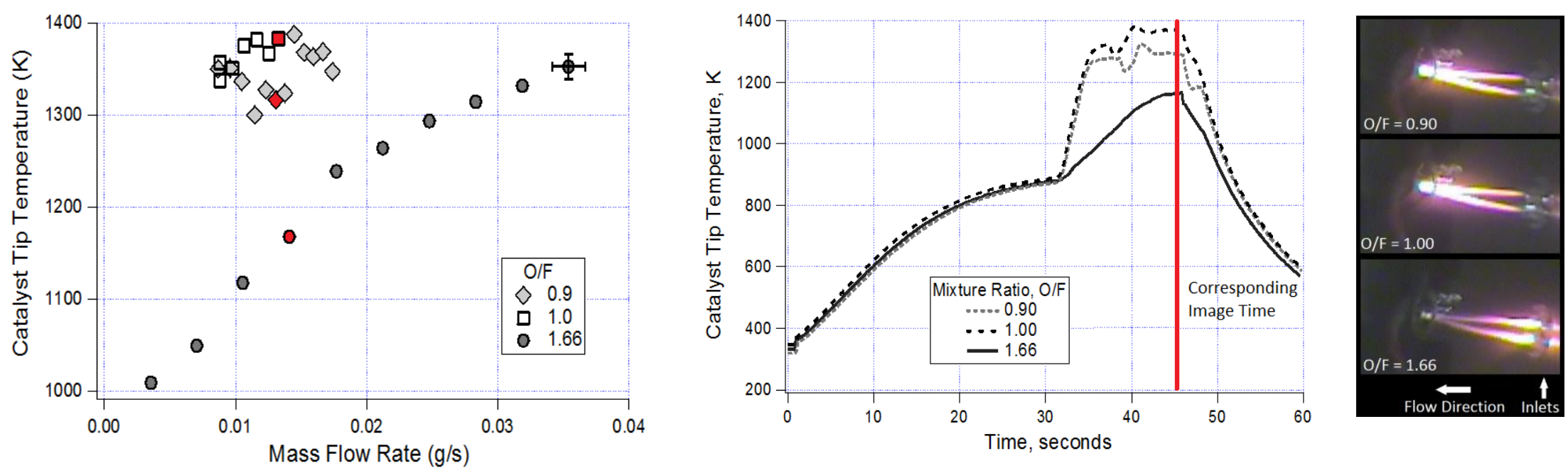

All cases at $8.1+/-0.2$ amps, Preheat $\mathrm{T} \sim 860 \mathrm{~K}$ 


\section{Staging}

- Main igniter flows on the order of $1.0 \mathrm{~g} / \mathrm{s}$

- Catalyst at $0.01 \mathrm{~g} / \mathrm{s}$ ( $1 \%$ of main flow), too low to ignite the main igniter flows unassisted

- Catalyst (low O/F) ignites a primary augmenter (high O/F) with similar order of magnitude flow (1\% of main flow)

- The $1^{\text {st }}$ stage flame ignites a secondary augmenter (nonpremixed low O/F, 10\% of main flow)

- Both stages used a manifold system with multiple impinging ports to inject and mix propellant flows
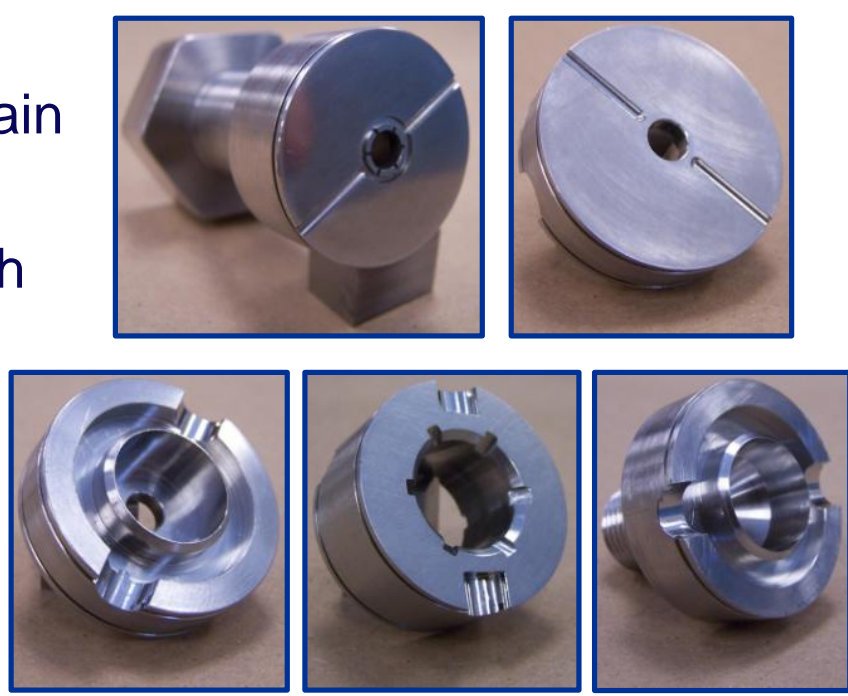

- Exit channel is threaded to match spark plug threads for comparable hardware
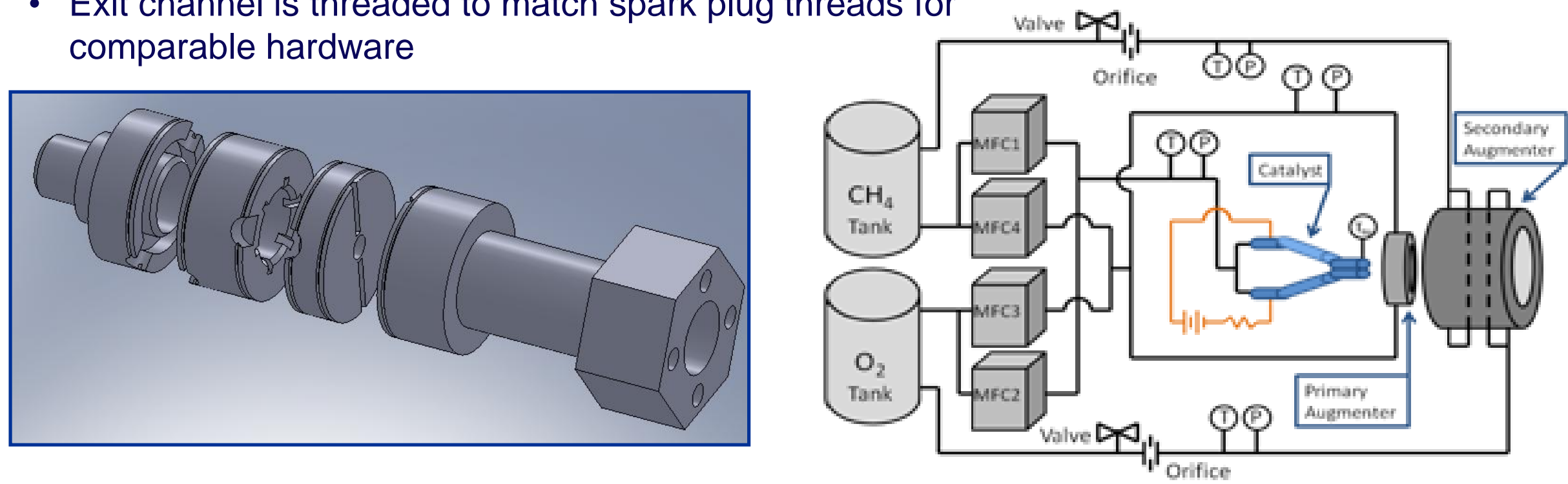


\section{Primary Augmenter Ignition}

- Initial testing at atmospheric confirmed ignition of primary augmenter:

- Flows from $0.005 \mathrm{~g} / \mathrm{s}$ to $0.05 \mathrm{~g} / \mathrm{s}$

- Mixture Ratio from 2.5 to 22.5 .

- Low O/F mixtures ( 2.5) demonstrated ignition but temperatures were lower than desired

- High O/F mixtures from 5.0 to 22.5 had catalyst tip temperatures from $1550 \mathrm{~K}$ to $1750 \mathrm{~K}$

- With limited time at altitude a few confirming cases (see graph) were run with variation in high O/F mixture ratio range and across nominal flow rate regime

- Ignition was achieved readily with current of >10 amps applied
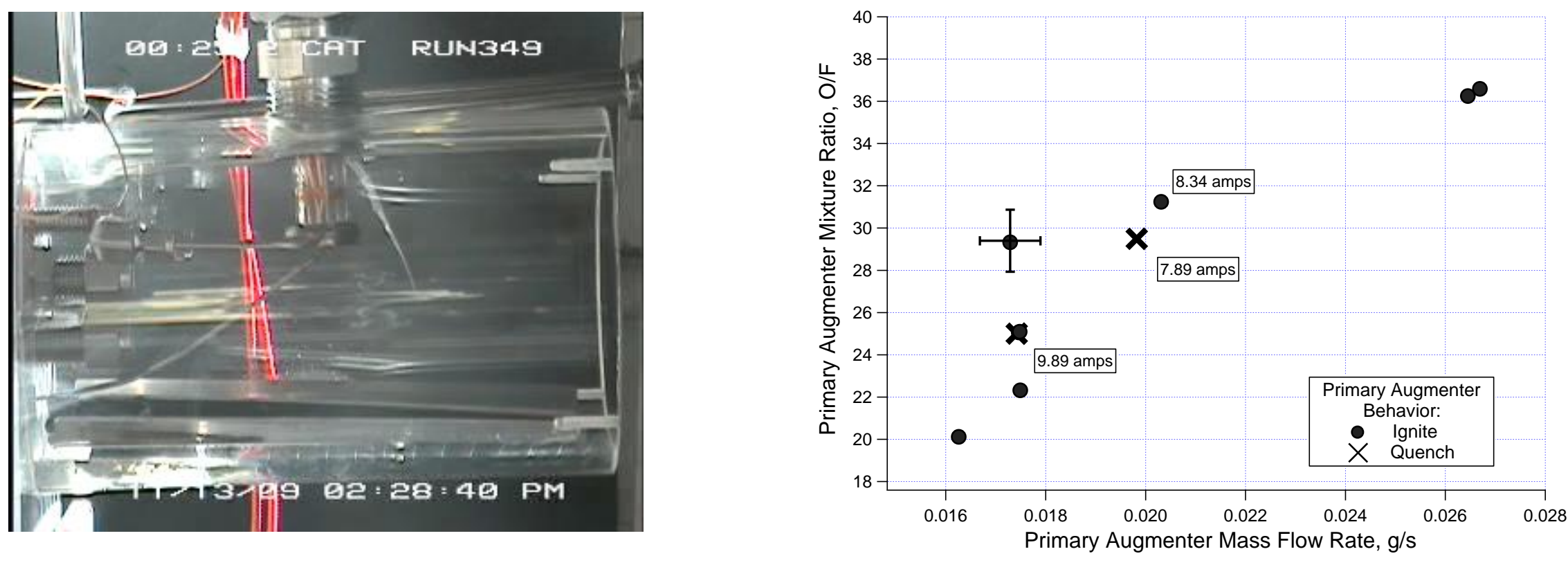


\section{Secondary Augmenter Ignition}

- Initial testing at atmospheric confirmed ignition of secondary augmenter:

- Flows from $0.15 \mathrm{~g} / \mathrm{s}$ to $0.45 \mathrm{~g} / \mathrm{s}$

- Mixture ratio from 1.0 to 2.0

- Testing at altitude (see graph) reconfirmed this regime, no mass flow or mixture ratio limits were observed within allowable variation

- Only two non-lights of the secondary augmenter occurred

- One below the required catalyst temperature ( 800 K)

- One below 10 amps, similar to the primary augmenter case
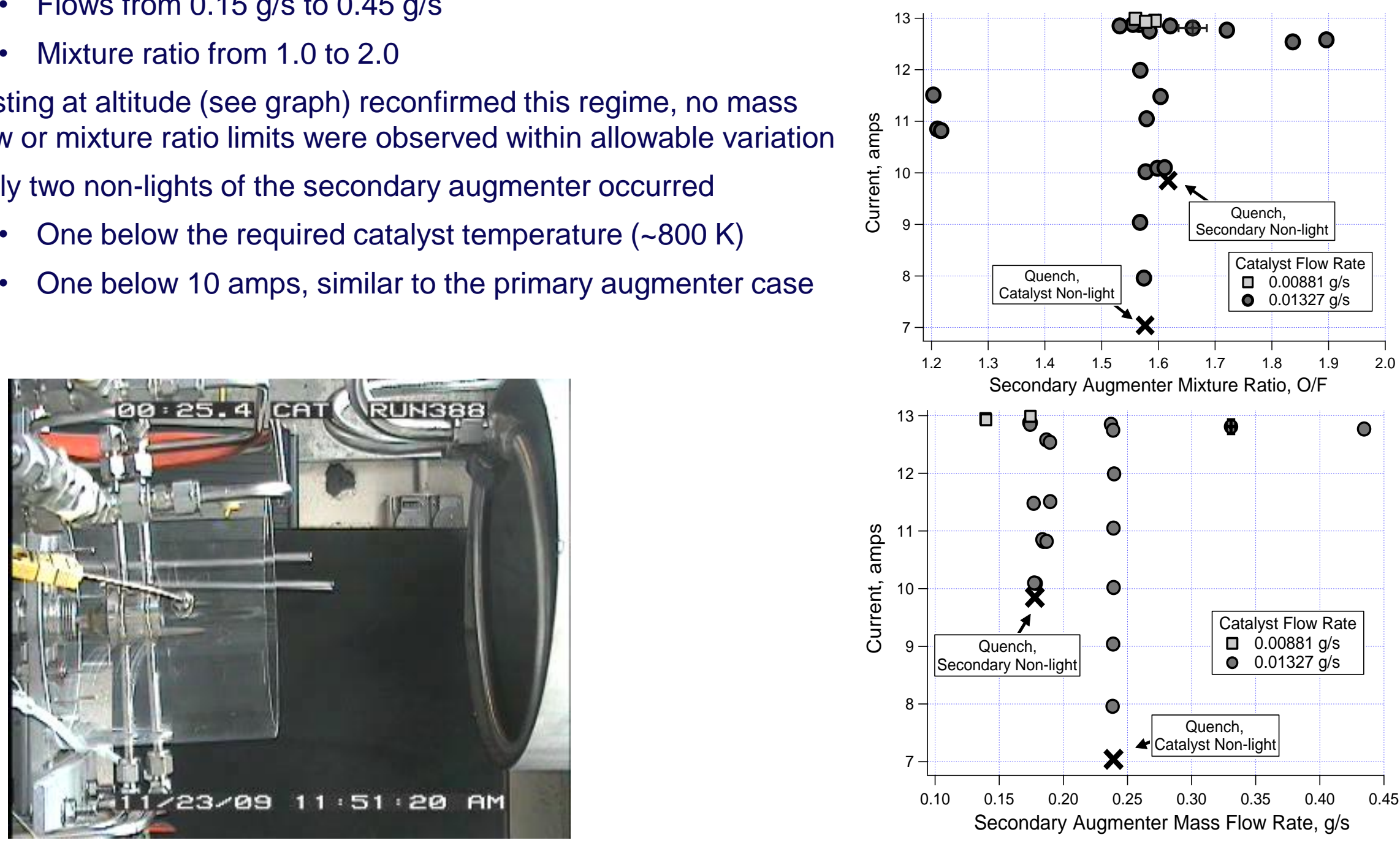


\section{Main Torch Ignition}

- Main igniter consists of two ports feeding opposing methane and oxygen flows sized for proper impingement

- Main flows are perpendicular to the staged flows

- Nozzle designed to choke with $\sim 1.0 \mathrm{~g} / \mathrm{s}$, pressurize to $150 \mathrm{psi}$ with $\sim 6.5 \mathrm{~g} / \mathrm{s}$

- Flow rate varied from $\sim 0.5 \mathrm{~g} / \mathrm{s}$ to $9.5 \mathrm{~g} / \mathrm{s}$, mixture ratio varied from 1.2 to 1.6

- Torch flame produced through range

- Low threshold quenching occurred with test points below $\mathrm{O} / \mathrm{F}=1.3$

- No mass flow rate limits observed within system variation allowable

- $\mathrm{C}^{\star}$ efficiency ranged from 90.5 to $95.5 \%$
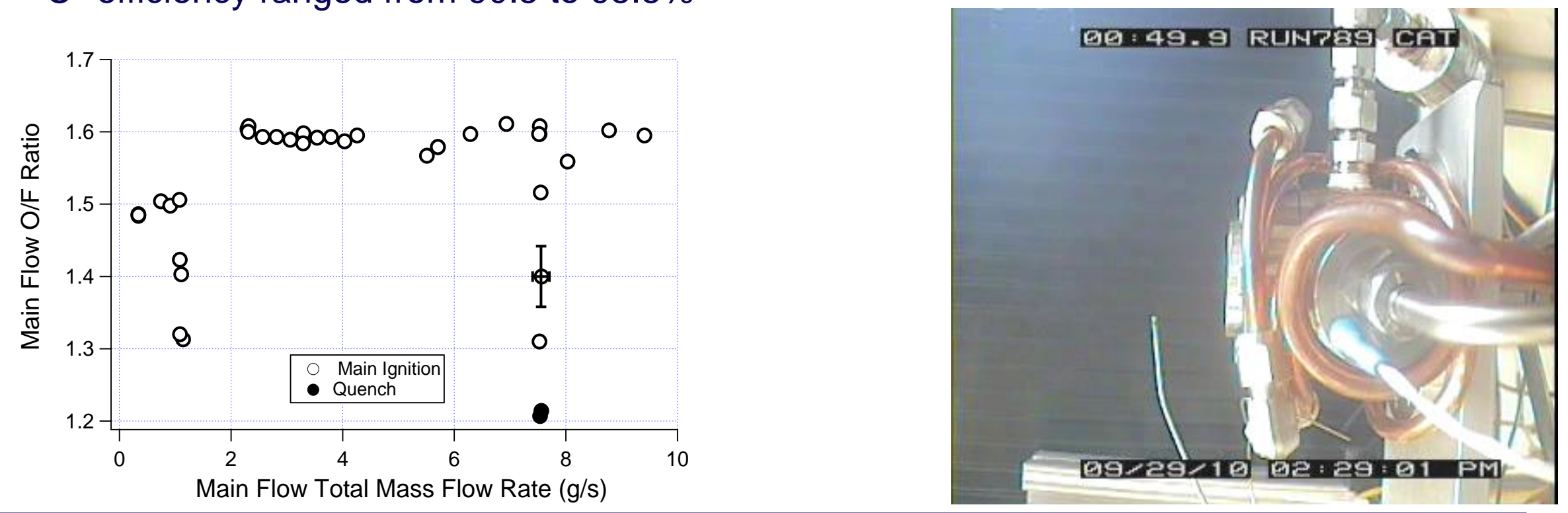


\section{Conclusions}

- This study developed an innovative microtube-based catalytic ignition system

- The unique design of the catalyst optimized the exit temperature of the vented combusted gas and this design is currently being pursued for a patent, NASA Glenn Research Center Case LEW-18565-1

- This system demonstrated and characterized staged ignition of flows by the catalytic microtubes at both atmospheric and altitude conditions

- This system can be utilized as a low power ignition source with required power to the catalyst of under $30 \mathrm{~W}$

- This is improvement over both comparable state of the art spark and alternative catalytic ignition systems

- Under atmospheric conditions, these stages were able to ignite as a spark plug replacement and generate torch ignition in a configuration comparable to the current SOA

- M. Deans thanks the NASA Graduate Student Researchers Program and the NASA Glenn Research Center Co-Op program for the support that made this effort possible. 


\section{Questions/Comments?}

